\title{
Clean Bulk YBaCuO Superconductors Doped by Paramagnetic Ions of Sm and $\mathrm{Yb}$
}

\author{
M. Jirsa ${ }^{a, b, *}$, D. Volochová ${ }^{c}$, J. KováC $\check{C}^{c}$ AND P. DikO ${ }^{c}$ \\ ${ }^{a}$ Institute of Physics, Czech Academy of Sciences, Na Slovance 2, CZ-18221 Praha 8, Czech Republic \\ ${ }^{b}$ Institute of Plasma Physics, Czech Academy of Sciences, Za Slovankou 3, CZ-18200 Praha 8, Czech Republic \\ ${ }^{c}$ Institute of Experimental Physics, SAS, Watsonova 47, 04001 Košice, Slovakia
}

\begin{abstract}
The recently developed clean cuprate technology was employed to study the effect of tiny amounts of paramagnetic $\mathrm{Yb}$ and $\mathrm{Sm}$ ions on electromagnetic properties of bulk $\mathrm{YBaCuO}$. Small samples of about $1.5 \times 1.5 \times 0.5 \mathrm{~mm}^{3}$ were measured by vibrating sample magnetometer in the field range $\pm 9 \mathrm{~T}$ and temperatures $30 \mathrm{~K}$ to $10 \mathrm{~K}$ in the non-superconducting tetragonal state and $300 \mathrm{~K}$ to $70 \mathrm{~K}$ in the orthorhombic, superconducting state. In the latter case, we observed the reversible paramagnetic moment above $T_{\mathrm{C}}$, similar as in the tetragonal state. Below $T_{\mathrm{C}}$ an irreversible magnetic moment was observed from which critical current density, $J_{c}$, was evaluated using the extended Bean model. The effect of doping by $\mathrm{Yb}$ and $\mathrm{Sm}$ ions on $J_{c}$ was studied and a correlation between $J_{c}$ and the total paramagnetic moment was found. Both $J_{c}(B)$ and the normalized pinning force density as a function of the reduced magnetic field were analyzed in terms of the classical model and discussed with respect to the material anisotropy, giving us at least a rough idea on the type of the effective pinning type and mechanism.
\end{abstract}

DOI: 10.12693/APhysPolA.131.1027

PACS/topics: 74.25.Ha, 74.25.Op, 74.25.Sv, 74.60.Ge, 74.72.--h

\section{Introduction}

It was realized relatively early $([1,2]$ and references therein) that practically all commercially available refractory and noble metals and their alloys used as a crucible materials for cuprate single crystal growth react intensively with the melts. Later it was found that yttrium-stabilized $\mathrm{ZrO}_{2}$ crucibles react with the melt so that an inert $\mathrm{BaZrO}_{3}$ compound forms, which does not further react with the melt and thus it represents an ideal material for inert crucibles $[3,4]$. Technology of melt-grown cuprates differs from that for single crystals. The solid pellet is melt only partially during the meltgrowth process and crucible is usually not necessary. It has been therefore believed that the cuprate material did not strongly interact with its surroundings. However, our recent study of paramagnetic moment of the light rare-earth (LRE) ions in (Y,LRE)- $\mathrm{Ba}_{2} \mathrm{Cu}_{3} \mathrm{O}_{y}$ compounds showed deviations from the expected behavior [5]. Their origin was identified as a contamination by $\mathrm{Yb}$ diffusing from the supporting Yb-based substrates and by Sm diffusing from Sm-123 seed [6]. This led to an improvement of the cuprate technology with the aim to fabricate clean, well defined superconducting structures. In the present work we report on the study of the electromagnetic characteristics of Y-123 bulks prepared in the new manner, doped in a controlled way by tiny amounts of Sm and $\mathrm{Yb}$.

\section{Experimental details}

YBCO single grain bulk superconductors with various concentrations of $\mathrm{Yb}_{2} \mathrm{O}_{3}$ and $\mathrm{Sm}_{2} \mathrm{O}_{3}$ were fabri-

\footnotetext{
*corresponding author; e-mail: jirsa@fzu.cz
}

cated by the top seeded melt growth process in air. To $1 \mathrm{~mol} \mathrm{YBa}_{2} \mathrm{Cu}_{3} \mathrm{O}_{7}$ powder, $0.25 \mathrm{~mol} \mathrm{Y}_{2} \mathrm{O}_{3}, 1 \mathrm{wt} \%$ $\mathrm{CeO}_{2}$, and $x^{\prime}\left(y^{\prime}\right)$ wt\% of $\mathrm{Yb}_{2} \mathrm{O}_{3}\left(\mathrm{Sm}_{2} \mathrm{O}_{3}\right)$ powder was added $\left(x^{\prime}, y^{\prime}=0.1 \div 1 \mathrm{wt} \%\right)$. We supposed that these values corresponded to: $x(y)=0.004 \div 0.02$ in the $\left(\mathrm{Y}_{1-x} \mathrm{Yb}_{x}\right)\left(\mathrm{Ba}_{1-y} \mathrm{Sm}_{y}\right)_{2} \mathrm{Cu}_{3} \mathrm{O}_{7-\delta}$ compositions.

Here we note that $\mathrm{Yb}$ is a heavy rare earth, substituting exclusively $\mathrm{Y}$ in the $\mathrm{YBaCuO}$ structure, while $\mathrm{Sm}$ is a light rare earth, going to both $\mathrm{Y}$ and Ba sites. Only the substitution of Sm for Ba was here considered.

The precursor powders were mixed for $30 \mathrm{~min}$ in a mixer and then intensively milled for $15 \mathrm{~min}$ in a friction mill. The mixed powders were uniaxially pressed into cylindrical pellets of $20 \mathrm{~mm}$ in diameter. The samples were treated in a chamber furnace with the time/temperature profile optimized for high Y-123 crystal quality [7]. Magnetic properties were tested on the samples cut from the pellet at the top surface, some $3 \mathrm{~mm}$ from the seed. They had in general dimensions $1.5 \times 1.5 \times 0.5 \mathrm{~mm}^{3}$. The measurements were conducted first in the tetragonal state, then, after annealing in oxygen for 1 week, in the orthorhombic state. Magnetization curves were measured by a vibrating sample magnetometer in magnetic fields up to $9 \mathrm{~T}$ at temperatures between $300 \mathrm{~K}$ and $10 \mathrm{~K}$ in the tetragonal state and between $300 \mathrm{~K}$ and $70 \mathrm{~K}$ in the orthorhombic state of the sample, with magnetic field lying in the sample plane.

A broader series of samples was tested at only one temperature, $77 \mathrm{~K}$, by SQUID with a vibrating sample mode option, with magnetic field up to $7 \mathrm{~T}$. Magnetic field was in this case oriented along $c$-axis.

\section{Experimental results}

In the first step the samples were measured in the tetragonal state showing only the reversible magnetic 
background. The measurements were conducted in a series of temperatures ranging from room temperature (RT, $300 \mathrm{~K}$ ) to $10 \mathrm{~K}$. The results are displayed in Fig. 1. At the lowest temperatures the paramagnetic $m(B)$ dependence is curved due to deviation from the Curie-Weiss approximation towards the full Brillouin function.

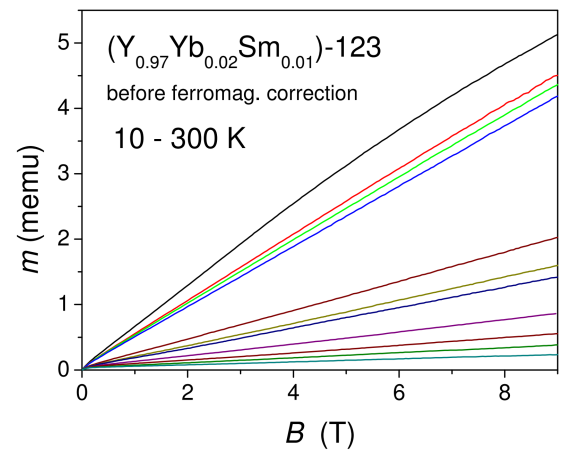

Fig. 1. Reversible magnetization loops of the tetragonal $\mathrm{YBaCuO}$ sample doped by $2 \% \mathrm{Yb}$ and $1 \% \mathrm{Sm}$ measured at 300,250,200,150,100,90,70,30,20,15, and $10 \mathrm{~K}$.

The curves for the highest temperatures, 250 and $300 \mathrm{~K}$ (Fig. 2) clearly showed a weak ferromagnetic component. It was modelled by a hyperbolic tangent function and subtracted from the total $m(B)$ dependence.

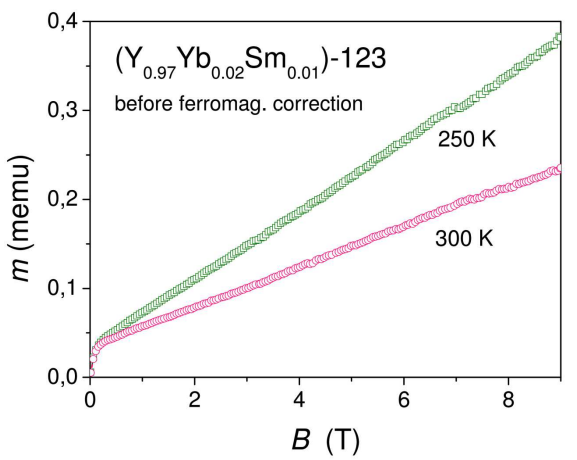

Fig. 2. The $m(B)$ dependences for 250 and $300 \mathrm{~K}$ show a weak, nearly reversible ferromagnetic contribution at low magnetic fields.

The inverse susceptibility of the paramagnetic background displayed as a function of temperature was used for evaluation of Weiss temperature, $\Theta$. Following the Curie-Weiss law, the $\chi^{-1}(T)$ dependence should be a straight line crossing $\chi^{-1}=0$ level at $T=\Theta$. The curvature of the $\chi^{-1}(T)$ dependence (Fig. 3, upper curve) revealed a significant diamagnetic contribution. After its correction the $\chi^{-1}(T)$ dependence became linear, extrapolating to $\Theta=-25 \mathrm{~K}$.

The two other samples, the non-doped Y-123 and the Y-123 doped by $1 \mathrm{~mol} \% \mathrm{Yb}$ and $2 \mathrm{~mol} \% \mathrm{Sm}$, exhibited in the tetragonal state qualitatively the same behavior.

After determining the background contributions, the samples were annealed in oxygen for about one week. After the annealing the samples became superconducting,

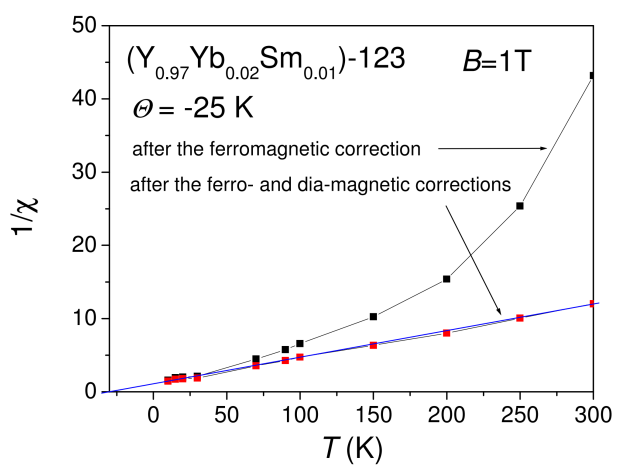

Fig. 3. Inverse susceptibility as a function of temperature; the upper curve before diamagnetic correction, the lower one after it.

orthorhombic, with critical temperature around $91 \mathrm{~K}$. Above $T_{\mathrm{C}}$, reversible paramagnetic dependences modified by tiny ferromagnetic contributions were observed, as before. Surprisingly, the diamagnetic component disappeared. We observed such a behavior also before, on similar samples prepared by the "dirty" technology. The Weiss temperature was for the orthorhombic sample from Fig. 3 nearly the same, $\Theta=-29.8 \mathrm{~K}$. The irreversible moment was much stronger than that of paramagnetic, diamagnetic, and ferromagnetic components altogether. For magnetic field within the sample plane, the magnetization curves are displayed in Fig. 4.

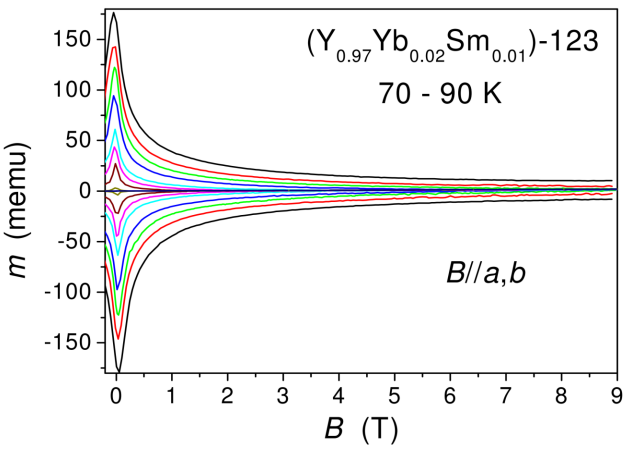

Fig. 4. Magnetic hysteresis loops for $\boldsymbol{B} \|(a, b)$-plane measured at temperatures $70,74,77,80,84,86,88$, $90 \mathrm{~K}$.

Using the extended Bean model, we determined the critical current density, $J_{c}$, as shown in Fig. 5. Again, the other two samples exhibited very similar behavior, only with somewhat lower critical current densities. To evaluate the effect of doping, four samples with different dopant concentrations (including that from Fig. 5) were tested by SQUID magnetometer with a vibrating sample option, at $77 \mathrm{~K}$, with magnetic field along $c$-axis. The $J_{c}$ values obtained from this experiment are shown in Fig. 6. They prove that $J_{c}$ grows with increase of paramagnetic moment. Note that $\mathrm{Yb}$ possesses about five times more Bohr magnetons per free ion than Sm.

The shapes of the dependences in Fig. 6, clearly differ from those in Fig. 5. Due to layered structure of cuprates, 


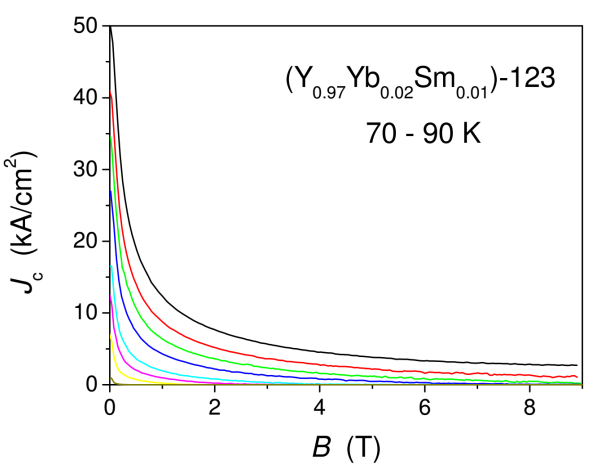

Fig. 5. Critical current densities as a function of magnetic field applied along the sample plane for temperatures $70,74,77,80,84,86,88,90 \mathrm{~K}$.

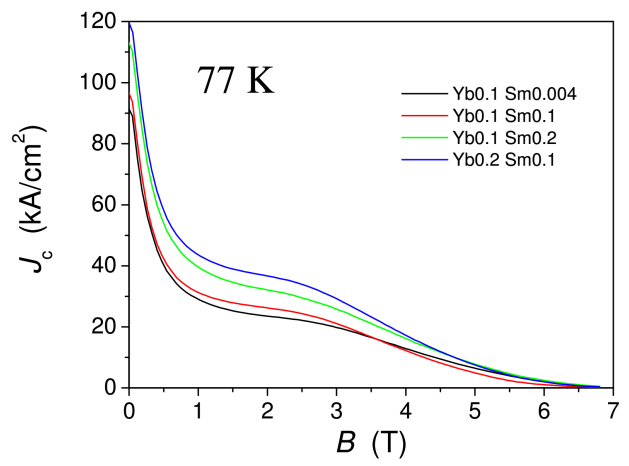

Fig. 6. Critical current densities as a function of magnetic field applied along $c$-axis for different amounts of dopants.

where only $\mathrm{CuO}_{2}$ planes are superconducting, the electric currents induced by a change of magnetic field are much higher in the $\boldsymbol{B} \| c$-axis case, where they flow exclusively within the superconducting planes, than with $\boldsymbol{B} \|(a, b)$-plane, where the currents flow in one direction within the $\mathrm{CuO}_{2}$ planes, but close the loops across the planes. With $\boldsymbol{B} \| c$-axis the vortices are Abrikosov-like,

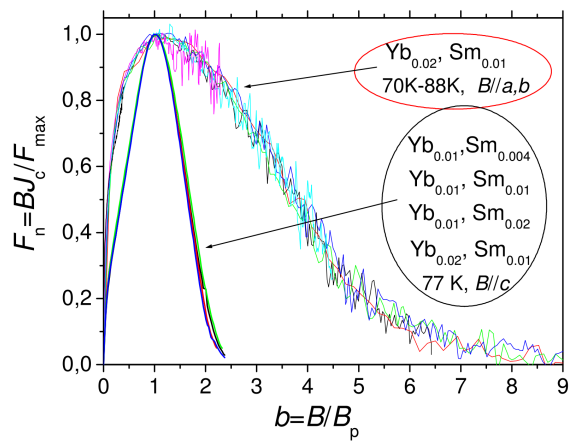

Fig. 7. Normalized pinning force density, $F_{n}=J_{c}^{*} B$, as a function of reduced magnetic field $b=B / B_{p}$. For $\boldsymbol{B} \|(a, b)$-plane the data were evaluated at $70,74,77$, $80,84,86$, and $88 \mathrm{~K}$. The irreversibility fields for $\boldsymbol{B} \| c$ axis were around $7.2 \mathrm{~T}$, for $\boldsymbol{B} \|(a, b)$-plane they ranged between $7 \mathrm{~T}(84 \mathrm{~K})$ and $2.6 \mathrm{~T}(88 \mathrm{~K})$. being rather firm stacks of round cross-section pancakes moving within $\mathrm{CuO}_{2}$ planes. With $\boldsymbol{B} \|(a, b)$-plane the vortices are more or less Josephson-like, of elliptical crosssection, and lie among the superconducting planes. No wonder that different pinning media are active in both cases and the shape of the hysteresis loop significantly differs. This is also documented by the graph of normalized pinning force density vs. reduced magnetic field (Fig. 7). It shows that the pinning mechanism in the $\boldsymbol{B} \| c$-axis configuration is practically unaffected by the $\mathrm{Yb}$ and Sm doping and in the $\boldsymbol{B} \|(a, b)$-plane configuration, at least in the Y-123 sample doped by $2 \mathrm{~mol} \% \mathrm{Yb}$ and $1 \mathrm{~mol} \% \mathrm{Sm}$, it is independent of temperature above $70 \mathrm{~K}$. Magnetic field orientation brings the principal difference; with $\boldsymbol{B} \| c$-axis a point-like pinning disorder responsible for the secondary peak in $J_{c}(B)$ prevails, shifting the $F_{n}(b)$ maximum towards the irreversibility field. In $\boldsymbol{B} \|(a, b)$-plane the intrinsic pinning by the individual oxide planes dominates, pushing the $F_{n}(b)$ maximum down to about one tenth of irreversibility field.

\section{Conclusions}

In the Y-123 bulk sample doped by $\mathrm{Yb}$ and $\mathrm{Sm}$ ions a reversible paramagnetic background was observed in the tetragonal, non-superconducting state, combined with a tiny ferromagnetic and diamagnetic signals. After annealing in flowing oxygen gas, the orthorhombic samples showed pure paramagnetic behavior only above $T_{\mathrm{C}}$; the diamagnetic signal disappeared. Striking differences in the $J_{c}(B)$ and $F_{n}(B)$ dependences were observed between magnetic field orientations along the $c$-axis and the $(a, b)$ plane, due to different effective pinning medium.

The main result of the work is the $J_{c}$ enhancement correlated with increase of total paramagnetic moment in the sample (amount of the $\mathrm{Yb}$ and $\mathrm{Sm}$ dopants).

\section{Acknowledgments}

This work was supported by the projects PhysNet, ITMS code 26110230097, APVV No. 0330-12, and VEGA No. 2/0121/16.

\section{References}

[1] A. Erb, T. Biernath, G. Müller-Vogt, J. Cryst. Growth 132, 389 (1993).

[2] A. Erb, T. Traulsen, G. Müller-Vogt, J. Cryst. Growth 137, 487 (1994).

[3] J.L. Zhang, J.E. Evetts, J. Mater. Sci. 29, 778 (1994).

[4] A. Erb, E. Walker, R. Flükiger, Physica C 245, 245 (1995).

[5] M. Jirsa, M. Rameš, M. Muralidhar, D. Volochová, P. Diko, Phys. Proced. 45, 65 (2013).

[6] D. Volochová, K. Jurek, M. Radusovska, S. Piovarci, V. Antal, J. Kovac, M. Jirsa, P. Diko, Physica C 496 , $14(2014)$

[7] D. Volochová, P. Diko, M. Radušovská, V. Antal, S. Piovarči, K. Zmorayová, M. Šefčiková, J. Cryst. Growth 353, 31 (2012). 